\title{
Ansiedade materna manifestada durante o tratamento odontológico de seus filhos
}

\author{
Laura Mendes Tomita - Universidade Estadual de Campinas \\ Áderson Luiz Costa Junior - Universidade de Brasília ${ }^{1}$ \\ Antônio Bento Alves de Moraes - Universidade Estadual de Campinas
}

\begin{abstract}
Resumo
A ansiedade manifestada pela criança durante o atendimento odontológico constitui um elemento potencialmente prejudicial à realização do tratamento bucal. $\mathrm{O}$ objetivo deste trabalho foi descrever e analisar os comportamentos de dez mães durante sessões seqüenciais de atendimento odontológico de seus filhos. Todas as sessões foram filmadas em videoteipe com marcas sonoras a cada 15 segundos, indicando os momentos em que foram efetuados os registros dos comportamentos. Um mapeamento geral do repertório de comportamentos permitiu apontar padrões comuns a todas as participantes e padrões específicos, indicadores de maior ansiedade de algumas participantes. Proporcionalmente, o procedimento de anestesia injetável evocou maior freqüência de comportamentos indicadores de respostas de ansiedade das mães. O cirurgião-dentista, atento aos comportamentos de pais e acompanhantes de crianças, pode adotar estratégias que reduzam a ansiedade dos pais, aumentando a freqüência de comportamentos colaborativos da criança com o tratamento.

Palavras-chave: Ansiedade em odontologia; Comportamentos de mãe; Análise de comportamentos.
\end{abstract}

\section{Maternal anxiety manifested during dental treatment of their children}

\begin{abstract}
The anxiety manifested by the child during the dentistry treatment constitutes a potentially harmful element to the accomplishment of the treatment. The aim of this study was to describe and analyse 10 mother's behavior during sequential sessions of their children's dental treatment. All sessions were recorded in videotape biped every 15 second interval, indicating the moments that the records of the behaviors would be carried out. A behavioral repertoire general plotting allowed to point common standards to all the participants and specific standards, larger anxiety indicators of some participants. Proportionally, the invasive anesthesia procedure evoked escape behaviors indicators larger frequency and/or mothers dodge. The pediatric dentistry, careful with parents' behaviors, can adopt strategies that reduce parents' anxiety, increasing behaviors frequency children's collaborating with the treatment.
\end{abstract}

Keywords: Mother's behavior; Dental anxiety; Behavior analysis.

\section{Introdução}

A Classificação Internacional de Doenças (CID10) caracteriza a ansiedade como um transtorno de caráter neurótico, freqüentemente relacionado a contextos de estresse e/ou somatoformes. Os sintomas, embora altamente variáveis, destacam elementos relacionados a: (a) apreensão - preocupações, sentimentos contínuos de nervosismo e pressentimentos; (b) tensão motora movimentação inquieta, tremores e incapacidade de relaxar; (c) hiperatividade autonômica - sensação de cabeça leve, sudorese, tonturas e cefaléias (OMS, 1993).

No contexto do atendimento odontológico, a ansiedade manifestada por crianças submetidas a tratamento bucal tem sido reconhecida como o principal

${ }^{1}$ Endereço para correspondência:

SQN, 206 - bloco G - apto 603 - 70844-070 - Brasília-DF

Telefone/Fax: (61) 3034-1685

E-mail: aderson@unb.br evento produtor de comportamentos não-colaborativos com a terapêutica (Aartman, van Everdingen, Hoogstraten \& Schuurs, 1998). No entanto, estudos recentes destacam que a maior parte dos comportamentos das crianças que parecem expressar ansiedade não são suficientemente compreendidos, ou são desconsiderados, por odontopediatras, constituindo-se em potenciais promotores de transtornos de comportamento que podem, inclusive, generalizar-se para outras situações de tratamento de saúde (Baier, Milgrom, Russell, Mancl \& Yoshida, 2004; Cardoso, Loureiro \& Nelson-Filho, 2004). Uma das implicações mais importantes desta situação é a diminuição da probabilidade de que a criança venha a colaborar com outros tratamentos a que for exposta, identificando essas situações como típicas de medo 
(Moraes, Possobon, Costa Junior \& Rolim, 2005).

Observa-se que, desde a década de 60 do século $\mathrm{XX}$, pesquisadores têm investigado a origem e $\mathrm{O}$ desenvolvimento da ansiedade no contexto do tratamento odontopediátrico. Embora não tenha sido possível identificar todas as variáveis relacionadas funcionalmente às manifestações de ansiedade de pacientes $\mathrm{e}$ acompanhantes, pode-se afirmar que sua ocorrência aponta para uma complexa etiologia multifatorial. Muitos autores têm atribuído os episódios de ansiedade infantil, na prática odontológica, à ansiedade demonstrada pela mãe que acompanha a criança ao consultório, apontando que essa relação pode resultar em maior probabilidade de comportamentos não-colaborativos da criança durante o atendimento em curso e em futuros tratamentos a que a criança vier a ser exposta (Allen \& Stokes, 1987; Johnson \& Baldwin, 1969; Klingberg \& Berggren, 1992; Kotsanos, Arhakis \& Coolidge, 2006; Milgrom, Mancl, King \& Weinstein, 1995; Ramos-Jorge, Pordeus, Serra-Negra \& Paiva, 1999; Wright, Alpern \& Leake, 1973).

Historicamente, o estudo de Johnson e Baldwin (1969) foi um dos primeiros a identificar uma correlação positiva e significativa entre a ansiedade materna e o repertório de comportamentos da criança em tratamento. Os participantes foram 60 crianças, de três a sete anos, cujo tratamento odontológico envolvia, pelo menos, uma extração dentária. Verificou-se que filhos de mães com elevado nível de ansiedade apresentavam taxas significativamente maiores de comportamentos concorrentes com o tratamento, quando comparadas às crianças com mães pouco ansiosas.

Wright e colaboradores (1973) investigaram os comportamentos de 124 crianças, de três a seis anos, expostas pela primeira vez a tratamento odontológico, bem como os indicadores comportamentais de ansiedade das mães. $\mathrm{O}$ objetivo era avaliar se uma explicação às mães sobre o procedimento a ser executado, antes do seu início, poderia reduzir a ansiedade materna, modificando o repertório de comportamentos das crianças. Os resultados mostraram que as mães que recebiam informações prévias evocavam, menos freqüentemente, comportamentos indicadores de ansiedade e foram mais eficientes na solicitação de colaboração das crianças, obtendo-se uma redução significativa dos comportamentos concorrentes das crianças em relação ao tratamento.

Allen e Stokes (1987) aplicaram técnicas de reforçamento positivo, por meio da liberação de pequenos brindes e elogios, contingentes a comportamentos colaborativos de crianças de três a seis anos, em tratamento odontológico. Além da redução nos níveis de não-colaboração com o tratamento, observou-se uma diminuição na freqüência de comportamentos indicadores de ansiedade, manifestados pelos pais que acompanhavam seus filhos ao consultório. Os autores sugerem que a redução na ocorrência de comportamentos não-colaborativos das crianças foi $\mathrm{o}$ resultado da combinação de efeitos da estratégia de reforçamento e da diminuição dos níveis de ansiedade e preocupação dos pais com o sofrimento da criança.

Klingberg e Berggren (1992) investigaram transtornos de saúde bucal e de comportamento em crianças cujos pais apresentavam níveis elevados de medo de dentista e recusa freqüente de se submeterem ao tratamento odontológico. Os resultados confirmaram a relação existente entre o medo dos pais e o medo apresentado por seus filhos na mesma situação. As crianças de pais com níveis mais altos de medo também tinham maiores índices de falta e cancelamento de consultas e maior probabilidade de abandonar $\mathrm{O}$ tratamento sem concluí-lo.

Kotsanos e colaboradores (2006) manipularam a presença e ausência de acompanhantes durante sessões de tratamento odontológico de crianças de três a oito anos, observando que a ausência dos pais constituía uma condição favorável ao aumento da freqüência de comportamentos colaborativos da criança às instruções do cirurgião-dentista. Pais ansiosos aumentavam a probabilidade de que seus filhos apresentassem comportamentos não-colaborativos com o tratamento.

No caminho oposto àqueles que destacam a relação entre os comportamentos dos pais e dos filhos no consultório odontológico, alguns estudos não identificam relação entre a ansiedade manifestada pelos pais, no consultório, e os comportamentos de seus filhos. Folayan, Adekoya-Sofowora, Otuyemi e Ufomata (2002), por exemplo, estudaram 81 crianças, de oito a treze anos, sem experiência odontológica. Os resultados mostraram que o nível de ansiedade das mães foi significativamente maior que o nível de ansiedade dos pais. No entanto, não foi identificada correlação estatisticamente significativa entre os níveis de ansiedade de mães e pais com comportamentos indicadores de ansiedade entre as crianças. Resultados semelhantes foram obtidos por Klaassen, Veerkamp e Hoogstraten (2003), bem como por Ten Berge, Veerkamp, Hoogstraten e Prins (2003).

Um aspecto crítico a ser considerado entre os trabalhos que se propõem a investigar a ansiedade materna no consultório de odontopediatria e sua relação com os comportamentos das crianças é a limitação metodológica no que se refere: (a) ao reduzido número de sessões experimentais, geralmente restritas a uma ou duas sessões, e ao fato de que nem sempre as sessões são analisadas em sua totalidade; (b) à grande quantidade de dados obtidos a partir da percepção dos pais sobre sua própria ansiedade, referida em entrevistas pré e/ou pós-

Psico-USF, v. 12, n. 2, p. 249-256, jul./ dez. 2007 
sessão e/ou por instrumentos de auto-avaliação, nem sempre validados; e (c) aos poucos estudos que utilizam metodologia observacional direta para identificar comportamentos indicadores de ansiedade e o contexto em que são evocados. Aponta-se a necessidade de se descrever e analisar funcionalmente o repertório de comportamentos das mães que acompanham seus filhos ao consultório de odontopediatria, antes de se investigar a possível relação entre esses comportamentos e as respostas apresentadas pelas crianças.

O objetivo deste trabalho foi descrever os comportamentos de mães que acompanham seus filhos ao consultório, durante sessões de tratamento odontológico, utilizando-se metodologia observacional direta.

\section{Metodologia}

\section{Participantes}

Os participantes foram 10 mães, que acompanhavam seus filhos, durante seis sessões seqüenciais de tratamento odontológico, ao Centro de Pesquisa e Atendimento Odontológico para Pacientes Especiais (Cepae) da Faculdade de Odontologia de Piracicaba (FOP), da Universidade Estadual de Campinas (UNICAMP).

Todas as 10 crianças tinham história de nãocolaboração com tratamento odontológico, conforme histórico registrado em serviços de odontologia da cidade e/ou região há, no máximo, seis meses. As crianças também tinham que apresentar um número mínimo de quatro molares cariados para que, da terceira à sexta sessão, um dente recebesse o tratamento necessário (de restauração). Com o intuito de padronizar o tratamento oferecido, além do número de dentes afetados, também foi considerada a gravidade da lesão cariosa, aceitando-se apenas crianças com lesões de cárie que necessitassem de anestesia local para o tratamento.

\section{Procedimento}

As duas primeiras sessões tinham objetivo de familiarizar a criança com o ambiente odontológico, sendo realizados apenas procedimentos de exame clínico e medidas profiláticas. Comportamentos colaborativos com o cirurgião-dentista eram seguidos de reforçamento positivo social. Comportamentos não-colaborativos com o cirurgião-dentista não eram seguidos de qualquer conseqüência. Nas quatro sessões seguintes, era realizado o tratamento odontológico curativo, sendo executadas as mesmas rotinas clínicas: exame clínico, anestesia tópica, anestesia injetável, isolamento absoluto, preparo cavitário, restauração e encerramento.

Todas as sessões foram integralmente gravadas em fitas de videoteipe com marcas sonoras, a cada 15 Psico-USF, v. 12, n. 2, p. 249-256, jul./ der. 2007 segundos, indicativas dos momentos em que se registrava a primeira ocorrência de cada categoria de comportamento previamente definida. Para a observação e registro dos comportamentos das mães, foram identificadas 16 categorias comportamentais, definidas operacionalmente e adaptadas com base no estudo de Possobon (2003):

- Acariciar/Tamborilar: passar levemente a palma, costas ou os dedos da mão sobre parte do rosto, cabelo ou corpo da criança ou tocar com a mão, rápida e sucessivamente, parte do corpo da criança, mantendo ritmo constante; ou, ainda, estando com a mão apoiada em parte do corpo da criança, movimentar suavemente a mão sem retirá-la do local.

- Beijar: tocar lábios unidos e protusos em alguma parte do corpo ou rosto da criança, com ou sem estalido.

- Consolar criança: tentar aliviar ou suavizar a aflição, o sofrimento da criança. Por exemplo: "não fique assim", "ô meu amor".

- Conter criança: Manter a(s) mão(s) parada(s), sobre ou em volta de parte(s) do corpo da criança, exercendo pressão e impedindo ou interrompendo seus movimentos, como flexão de pernas e braços e toque no profissional ou em objetos utilizados no procedimento.

- Desviar olhar: Imediatamente antes ou durante a inserção da agulha no corpo da criança, fechar os olhos, podendo fazer movimentos de pressão da pálpebra superior sobre a inferior; virar a cabeça para o lado oposto ao procedimento realizado ou abaixar a cabeça, olhando em outra direção.

- Distrair criança: dirigir à criança uma frase afirmativa ou interrogativa ou conversar sobre assuntos não relacionados ao procedimento ou cantar e tentar desviar a atenção da criança do procedimento que está sendo realizado.

- Instruir criança: dizer para a criança o que ela deve fazer. Por exemplo: "abra a boca", "coloque as mãos para baixo", "não se mexa".

- Lamentar: manifestar, por meio de palavras, o seu sofrimento. Por exemplo: "ai, meu Deus do céu". Queixa, acompanhada de gemidos, lamúria.

- Observar procedimento: olhar atentamente em direção ao procedimento que está sendo realizado na criança.

- Oferecer ajuda: manifestar verbalmente apoio à criança. Por exemplo: "a mamãe está aqui com você".

- Pegar no colo: suspender a criança até a altura do tronco, segurando seu corpo ou parte dele, e aproximá-la de si, 
de modo que esta fique sustentada pelos braços e apoiada em parte do corpo da mãe.

- Posicionar criança: segurando o corpo da criança em qualquer posição inicial, colocá-la sobre uma superfície, de modo que todo o seu corpo fique em contato com esta, ou mudar a criança de posição e acomodá-la sobre a cadeira odontológica, de modo a facilitar a atuação do profissional.

- Prometer recompensa: assegurar à criança a ocorrência de um evento agradável, após a realização do procedimento.

- Questionar procedimento: questionar o profissional em relação a algum aspecto do procedimento.

- Seguir instrução: atender solicitação do profissional, como conter a criança ou conversar com ela.

- Segurar mão: encostar a(s) palma(s) da(s) mão(s) na mão da criança, flexionando os dedos em torno dela.

Os registros foram realizados por dois observadores independentes, previamente treinados, que assistiam, separadamente, às sessões gravadas e efetuavam o registro dos comportamentos das mães, indicando-se, ainda, a rotina clínica em execução. Os registros foram, então, comparados e verificou-se índice de concordância, entre observadores, superior a $85 \%$.

O projeto de pesquisa foi submetido e aprovado pelo Comitê de Ética em Pesquisa da FOP-UNICAMP. Os dados apresentados neste trabalho referem-se apenas às sessões nas quais foram executados procedimentos odontológicos curativos.

\section{Resultados}

As categorias de comportamento acariciar/ tamborilar, conter criança, instruir criança, observar procedimento, posicionar criança e segurar mão foram comuns a todas as participantes.
A categoria acariciar/tamborilar, apresentada por todas as participantes, foi observada com maior freqüência na rotina odontológica de anestesia injetável, seguida pela anestesia tópica e preparo cavitário, destacando-se que a freqüência de ocorrência durante a anestesia injetável foi $68 \%$ maior do que a registrada na anestesia tópica e $119 \%$ maior do que a registrada no preparo cavitário.

Já a categoria observar procedimento, também apresentada por todas as mães, teve freqüência de ocorrência $176 \%$ maior que a registrada para a categoria acariciar/tamborilar. A maior freqüência ocorreu na rotina de preparo cavitário, seguida da anestesia injetável e anestesia tópica.

Somente M2 apresentou a categoria lamentar. Já a categoria questionar procedimento foi observada em M2 e M10. A categoria desviar olhar apresentou as maiores freqüências e variabilidades ao longo das sessões de atendimento, também para M2 e M10. Essas mães foram as que demonstraram maior variabilidade comportamental quanto a ocorrência e freqüência de comportamentos durante as quatro sessões de observação.

No outro extremo, identificou-se M3, participante que apresentou o repertório comportamental menos variado ao longo das sessões, sendo a única a não apresentar as categorias desviar olhar e oferecer ajuda, registradas em todas as outras mães. As demais participantes (M1 e M4 a M9) apresentaram padrões de comportamento semelhantes (indicados por - na Figura 1), o que sugere que este seja um padrão comportamental adotado pela maioria das mães durante $\mathrm{o}$ atendimento odontológico de seus filhos.

A Figura 1 ilustra o aumento gradual, ao longo das sessões de tratamento curativo, da freqüência de ocorrência da categoria segurar mão, que variou de $3,6 \%$ na terceira sessão para $34 \%$ na sexta sessão, ao mesmo tempo em se observava uma redução da freqüência de ocorrência da categoria conter criança.
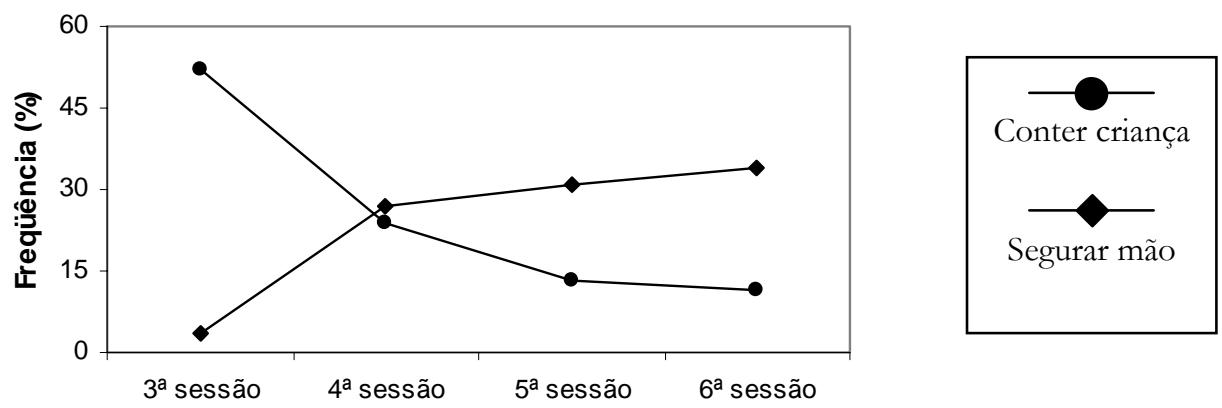

Figura 1 - Freqüência de ocorrência das categorias de comportamento conter criança e segurar mão ao longo das quatro sessões de tratamento de todas as participantes 
A Figura 2 apresenta a freqüência de ocorrência da categoria desviar olhar, ao longo de cada rotina odontológica, durante as quatro sessões de tratamento curativo. Pôde-se notar que as maiores freqüências de ocorrência dessa categoria ocorreram durante os procedimentos de anestesia injetável e isolamento absoluto.

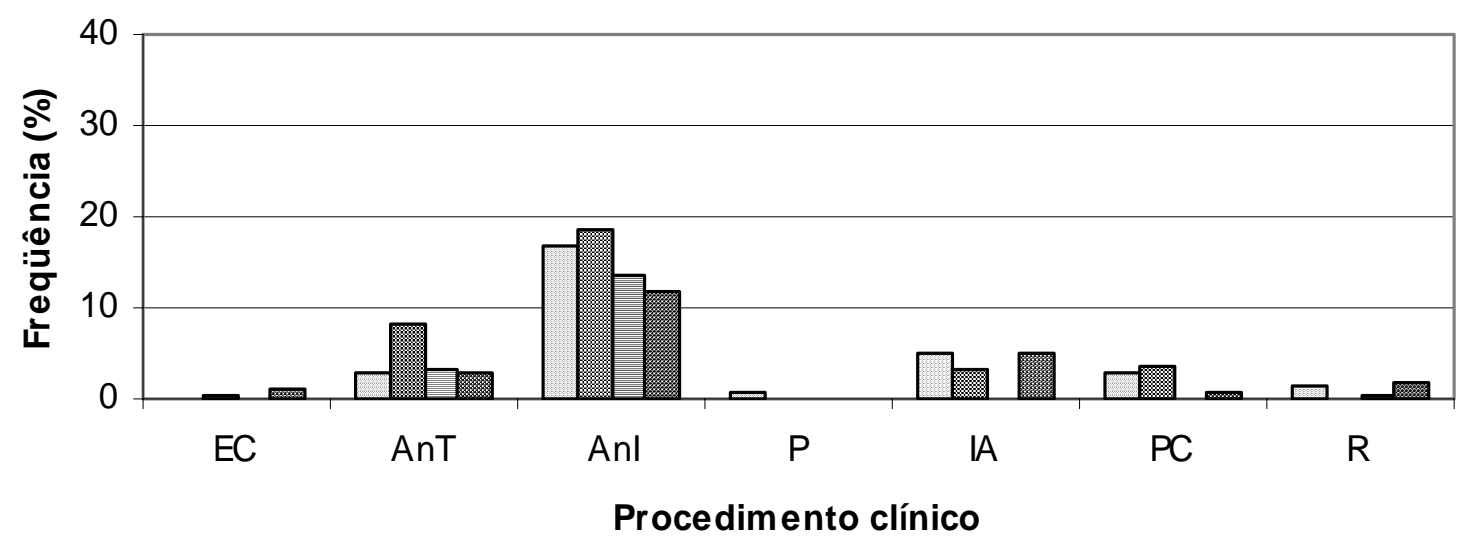

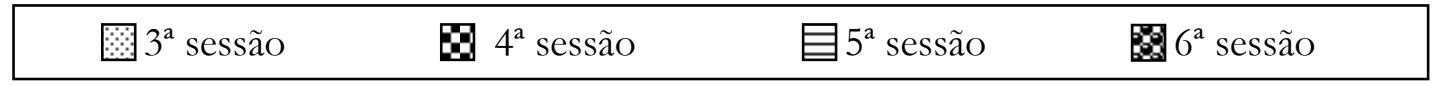

EC: exame clínico; AnT: anestesia tópica; AnI: anestesia injetável; P: pausa; LA: isolamento absoluto; PC: preparo cavitário; R: restauração.

Figura 2 - Freqüência de ocorrência da categoria de comportamento desviar olhar, ao longo de cada procedimento clínico, considerando-se todas as participantes

\section{Discussão}

Os comportamentos das mães relacionados a posicionar criança, instruir criança e conter criança são, muitas vezes, essenciais para que o atendimento odontológico prossiga e, por isso, fizeram parte do repertório comportamental de todas as mães estudadas.

Dentre as categorias de comportamento apresentadas por todas as participantes, pode-se destacar as categorias acariciar/tamborilar e observar procedimento. As maiores freqüências foram registradas nas rotinas de anestesia e preparo cavitário (para acariciar/tamborilar) e, em ordem inversa, para observar procedimento, rotinas que envolvem a inserção de agulha e broca de alta rotação e que são identificadas como invasivas. Apesar de o procedimento de registro observacional adotado no estudo caracterizar-se pela obtenção de dados por amostragem, a alta freqüência de ocorrência da categoria observar procedimento, concomitante à ocorrência da categoria acariciar/ tamborilar, permite afirmar que a maior parte das mães que acariciavam seus filhos não estavam olhando para eles, mas para o procedimento odontológico em execução. Levanta-se a hipótese de que a disponibilização de informação prévia sobre as rotinas odontológicas a serem executadas, incluindo suas características conforme a condição bucal de cada criança, poderia aumentar a probabilidade de que as mães acariciassem seus filhos olhando para eles e não para o procedimento odontológico, caracterizando uma condição afetiva mais reforçadora à criança.

M2 e M10 foram consideradas as mães com maior freqüência de comportamentos indicadores de ansiedade, expresso em respostas que indicavam percepção de sofrimento e/ou necessidade de buscar mais informações por meio de questionamentos ao cirurgião-dentista. O repertório dessas mães foi coerente com o descrito pela CID-10 como sintomas indicadores de ansiedade em situações específicas, que incluem ampla variabilidade comportamental, em termos motores, e que denotam nervosismo, tensão muscular, desconforto físico e psicológico e preocupações (OMS, 1993).

$\mathrm{O}$ fato de comportamentos possivelmente indicadores de ansiedade terem sido identificados particularmente em apenas duas participantes pode estar relacionado com as duas sessões iniciais de atendimento, nas quais não ocorriam procedimentos curativos. Tais sessões foram utilizadas para familiarizar as crianças e as mães com o ambiente físico do consultório, os equipamentos e o cirurgião-dentista, sendo realizados, apenas, procedimentos profiláticos e não-invasivos. Sessões preparatórias têm sido utilizadas, em diversos estudos, com objetivo de reduzir a aversividade percebida em relação a ambientes de tratamento médico e odontológico (Bragado \& Fernandéz, 1997; Costa Junior, 2001; Possobon, 2003). 
O repertório comportamental pouco variável de M3 poderia indicar, aparentemente, uma mãe apática e alheia aos acontecimentos do ambiente ao seu redor, incluindo os procedimentos odontológicos que estavam sendo executados na criança. A análise dos comportamentos de M3, no entanto, não confirmariam esta hipótese. Destaca-se que a menor variabilidade de respostas de M3 não implicou em baixa freqüência de respostas. Esta participante mostrou-se extremamente atenta à necessidade de conter fisicamente a criança durante os episódios de não-colaboração com o tratamento. A preocupação em garantir que a criança apresentasse um mínimo de movimentos físicos, compatível com a perspectiva de "atrapalhar" o menos possível a atuação do profissional, agilizando a sessão e diminuindo o tempo de sofrimento de seu filho, tal como descreve Klatchoian (2002), poderia ser atribuída a M3.

A diminuição gradual, da terceira para a sexta sessão, da categoria conter criança (Figura 2) sugere um decréscimo da freqüência de comportamentos de nãocolaboração das crianças ao longo das sessões de atendimento. Allen e Stokes (1987), Stark e colaboradores (1989), bem como Ten Berge, Veerkamp e Hoogstraten (1999), observam que a redução da necessidade de contenção física da criança, ao longo de sessões de atendimento odontológico, está diretamente relacionada ao aumento dos níveis de colaboração da criança.

A diminuição da freqüência da categoria conter criança e o aumento da freqüência da categoria segurar mão sugerem que a categoria segurar mão poderia ser considerada uma expressão comportamental de apoio à criança, pois a mãe demonstra a sua presença ao lado do filho, segurando sua mão e incentivando-o a enfrentar o tratamento com maior eficiência (Costa Junior, 2001). Para Blount, Sturges e Powers (1990), observa-se que uma redução na ocorrência de comportamentos nãocolaborativos por parte de crianças submetidas a sessões consecutivas de procedimentos invasivos geralmente é seguida pelo aumento de comportamentos indicadores de carinho e atenção dos acompanhantes que presenciam a execução dos procedimentos invasivos. No mesmo sentido, um aumento da freqüência de carinho e atenção por parte dos pais presentes à execução de um procedimento invasivo em um dos filhos pode constituir um indicador de controle emocional e promotor de estratégias de enfrentamento mais eficientes e focalizadas no evento em andamento (Costa Junior, 2005).

A maior freqüência de ocorrência da categoria desviar olhar durante a execução da rotina de anestesia injetável (Figura 2) sugere que esta resposta esteja relacionada à percepção de aversividade do procedimento e/ou à crença da mãe quanto ao sofrimento que tal procedimento possa proporcionar à criança. Este resultado é coerente com o obtido pelo estudo de Possobon (2003), no qual a anestesia injetável foi a rotina que gerou maior nível de ansiedade nas crianças. Também para Cardoso (2002), a aplicação da anestesia injetável, a execução de extração/cirurgia dentária e o uso do motor de alta rotação (durante a rotina de preparo cavitário) foram os procedimentos mais freqüentemente considerados como de difícil enfrentamento para as crianças, sendo percebidos como ameaçadores ou incômodos pelos pais.

O comportamento das mães, de desviar olhar, especialmente durante a execução da anestesia injetável, pode ser analisado, também, como uma resposta de fuga, alternativa adotada pelas participantes para "evitar" o próprio sofrimento. Além disso, estudos que investigam os aspectos mais aversivos da situação odontológica encontraram índices elevados de não-colaboração das crianças durante os procedimentos de anestesia injetável (Ram, Mamber, Chosack \& Fuks, 1999; Yanase, Braham, Fukuta \& Kurosu, 1996).

A ocorrência de comportamentos como desviar olhar, questionar procedimento e lamentar, por exemplo, poderia constituir estímulos discriminativos para que o cirurgião-dentista disponibilizasse informação e esclarecimentos, permitindo que pais e acompanhantes expressassem suas preocupações e se tornassem participantes mais ativos do processo de tratamento odontológico de seus filhos. Ramos, Carrara e Gomide (2005), por exemplo, destacam a necessidade de que o cirurgião-dentista informe, previamente, aos pais e/ou acompanhantes, sobre procedimentos odontológicos e técnicas de manejo comportamental que poderão ser utilizados durante o atendimento à criança. Tal cuidado pode reduzir, significativamente, a apreensão dos pais acerca de procedimentos invasivos aos quais seus filhos poderão ser expostos.

Em outro estudo, Hicks, von Baeyer e McGrath (2005) destacam que os profissionais de saúde devem dispor de estruturas dinâmicas de intervenção que garantam cuidados psicossociais junto aos cuidados médicos e/ou odontológicos. Por meio de disponibilização de informações online sobre manejo da dor, os autores observaram um aumento da eficácia do tratamento prestado, bem como redução em indicadores de ansiedade e melhora em indicadores de qualidade de vida de pacientes e seus familiares.

Com base nos dados obtidos neste estudo, considerando-se que o comportamento de desviar olhar pode indicar uma condição de apreensão da mãe e aumentar a probabilidade de uma resposta de nãocolaboração da criança com o tratamento, a adoção de estratégias educativas pelo cirurgião-dentista, tal como proposto por Ramos e colaboradores (2005), poderia criar

Psico-USF, v. 12, n. 2, p. 249-256, jul./ dez. 2007 
uma condição de tratamento na qual a mãe não tivesse motivo para desviar o olhar da criança durante a execução de procedimentos clínicos considerados mais aversivos.

A realização deste estudo permitiu a descrição do repertório comportamental de que acompanham seus filhos durante sessões de tratamento odontológico. Pôdese notar que algumas mães demonstram maior nível de ansiedade do que outras, mas existem certos comportamentos que são comuns a todas elas. Segundo Borges (1999), cada mãe possui um padrão pessoal de responder a situações estressantes, adquirido ao longo de sua história de vida e mantido por inúmeros fatores ambientais, o que permite sugerir que os odontopediatras atentos aos comportamentos de pais e acompanhantes podem auxiliá-los a atuar como agentes estimuladores de comportamentos colaborativos das crianças em tratamento.

\section{Referências}

Aartman, I. H. A., van Everdingen, T., Hoogstraten, J. \& Schuurs, A. H. B. (1998). Self-report measurements of dental anxiety and fear in children: A critical assessment. Journal of Dentistry for Children, 65(4), 252-258.

Allen, K. D. \& Stokes, T. F. (1987). Use of escape and reward in the management of young children during dental treatment. Journal of Applied Behavior Analysis, 20(4), 381-390.

Baier, K., Milgrom, P., Russell, S., Mancl, L. \& Yoshida, T. (2004). Children's fear and behavior in private dentistry practices. Paediatric Dentistry, 26(4), 316-321.

Blount, R. L., Sturges, J. W. \& Powers, S. W. (1990). Analysis of child and adult behavioral variations by phase of medical procedure. Behavior Therapy, 21(1), 33-48.

Borges, L. M. (1999). Treinamento de respostas de enfrentamento e colaboração em mães de crianças submetidas a procedimentos médicos invasivos. Dissertação de Mestrado. Brasília, DF: Universidade de Brasília.

Bragado, C. A. \& Fernandéz, A. M. (1997). Psychological treatment of evoked pain and anxiety by invasive medical procedures in pediatric oncology. Spain Journal of Psychology, 1(1), 17-36.

Cardoso, C. L. (2002). Tratamento odontopediátrico no contexto de uma clínica-escola: avaliação do estresse da criança, do acompanhante e do aluno. Tese de Doutorado. Ribeirão Preto, SP: Universidade de São Paulo.

Cardoso, C. L., Loureiro, S. R. \& Nelson-Filho, P. (2004). Pediatric dental treatment: Manifestations of stress in patients, mother and dental school students. Pesquisa Odontológica Brasileira, 18(2), 150-155.

Costa Junior, A. L. (2001). Análise de comportamentos de crianças expostas à punção venosa para quimioterapia. Tese de Doutorado. Brasília, DF: Universidade de Brasília.

Costa Junior, A. L. (2005). Psicologia da saúde e desenvolvimento humano: o estudo do enfrentamento em crianças com câncer e expostas a procedimentos médicos invasivos. Em M. A. Dessen \& A. L. Costa Junior (Orgs.). A ciência do desenvolvimento bumano: tendências atuais e perspectivas futuras (pp. 171-189). Porto Alegre: Artes Médicas.

Folayan, M. O., Adekoya-Sofowora, C. A., Otuyemi, O. D. \& Ufomata, D. (2002). Parental anxiety as a possible predisposing factor to child dental anxiety in patients seen in a suburban dental hospital in Nigeria. International Journal of Paediatric Dentistry, 12(4), 255-259.

Hicks, C. L., von Baeyer, C. \& McGrath, P. J. (2005). Online psychological treatment for pediatric recurrent pain: A randomized evaluation. Journal of Pediatric Psychology Advance Access Published, 10, 1-13.

Johnson, R. \& Baldwin, D. C. (1969). Maternal anxiety and child behavior. Journal of Dentistry for Children, 36(2), 87-92.

Klaassen, M., Veerkamp, J. \& Hoogstraten, J. (2003). Predicting dental anxiety. The clinical value of anxiety questionnaires: An explorative study. European Journal of Paediatric Dentistry, 4(4), 171-176.

Klatchoian, D. (2002). Psicologia odontopediátrica. São Paulo: Ed. Santos.

Klinberg, G. \& Berggren, U. (1992). Dental problem behaviors in children of parents with severe dental fear. Sweden Dentistry Journal, 16(1/2), 27-32.

Kotsanos, N., Arhakis, A. \& Coolidge, T. (2006). Parental presence versus absence in the dental operatory: A technique to manage the uncooperative child dental patient. European Journal of Paediatric Dentistry, 6(3), 144148.

Milgrom, P., Mancl, L., King, B. \& Weinstein, P. (1995). Origins of childhood dental fear. Behavior Research and Therapy, 33(3), 313-319.

Moraes, A. B. A., Possobon, R. F., Costa Junior, A. L. \& Rolim, G. S. (2005). Contingências aversivas em serviços de saúde. Em H. J. Guilhardi \& N. C. de Aguirre (Orgs.). Sobre comportamento de cognição: expondo a variabilidade (pp. 83-94). Santo André, SP: Esetec.

OMS - Organização Mundial da Saúde (1993). Classificação de transtornos mentais e de comportamento da CID-10. Porto Alegre: Artes Médicas. 
Possobon, R. F. (2003). Efeitos do diazepam sobre os comportamentos não-colaborativos de crianças em atendimento odontológico. Tese de Doutorado. Piracicaba, SP: Universidade Estadual de Campinas.

Ram, D., Mamber, E., Chosack, A. \& Fuks, A. B. (1999). The effect of metoclopramide and hydroxyzine in sedation of infants undergoing dental treatment. Journal of Dentistry for Children, 66(1), 49-52.

Ramos, M. M., Carrara, C. F. \& Gomide, M. R. (2005). Parental acceptance of behavior management techniques for children with clefts. Journal of Dentistry for Children (Chicago), 72(2), 74-77.

Ramos-Jorge, M. L., Pordeus, I. A., Serra-Negra J. M. C. \& Paiva, S. M. (1999). A ansiedade materna como fator de influência na adaptação comportamental do paciente odontopediátrico. Arquivos de Odontologia, 35(1/2), 61-70.

Stark, L. J., Allen, K. D., Hurst, M., Nash, D. A., Rigney, B. \& Stokes, T. F. (1989). Distraction: Its utilization and efficacy with children undergoing dental treatment. Journal of Applied Behavior Analysis, 22(3), 297-307.

Sobre os autores:

Laura Mendes Tomita é cirurgiã-dentista, doutoranda do Programa de Pós-Graduação em Odontologia - área de Farmacologia, Anestesiologia e Terapêutica, na Faculdade de Odontologia de Piracicaba da Universidade Estadual de Campinas - Unicamp.

Áderson Luiz Costa Júnior é psicólogo, doutor em Psicologia e professor adjunto do Instituto de Psicologia da Universidade de Brasília - UnB. Área: Psicologia da Saúde e Desenvolvimento Humano.

Antônio Bento Alves de Moraes é psicólogo e cirurgião-dentista, doutor em Psicologia e professor titular da Área de Psicologia Aplicada na Faculdade de Odontologia de Piracicaba da Universidade Estadual de Campinas Unicamp.
Ten Berge, M., Veerkamp, J. S. \& Hoogstraten, J. (1999). Dentists' behavior in response to child dental fear. Journal of Dentistry for Children, 66(1), 36-40.

Ten Berge, M., Veerkamp, J. S., Hoogstraten, J. \& Prins, P. J. (2003). Childhood dental fear in relation to parental child-pearing attitudes. Psychological Reports, 92(1), 43-50.

Wright, G. Z., Alpern, G. D. \& Leake, J. L. (1973). The modifiability of maternal anxiety as it relates to children's cooperative dental behavior. Journal of Dentistry for Children, 40(4), 265-271.

Yanase, H., Braham, R. L., Fukuta, O. \& Kurosu, K. (1996). A study of the sedative effect of homeadministered oral diazepam for the dental treatment of children. Internartional Journal of Paediatric Dentistry, 6(1), 13-17.

Recebido em junho de 2006 Reformulado em abril de 2007 Aprovado em maio de 2007 